\title{
Commons without Tragedy: Sampling Dynamics and Cooperative Resource Extraction*
}

\author{
Juan Camilo Cárdenas ${ }^{\dagger} \quad$ César Mantilla Rajiv Sethi $^{\ddagger}$
}

July 31, 2013

\begin{abstract}
This paper reconsiders evidence from experimental common pool resource games from the perspective of a dynamic model of sampling. Despite being parameter-free, the model is able to replicate some striking features of the data: monotonic frequency distributions, the persistent use of strictly dominated actions, and stable heterogeneity in choices. We argue that these patterns cannot be fully accounted for by existing theories based on other-regarding preferences and norms, and that the dynamics of sampling provide a useful complementary explanation for behavior in social dilemmas.
\end{abstract}

Keywords: Common Pool Resources; Experiments; Sampling Equilibrium

JEL Classification Codes: JEL codes: C73, C91, D03, H41, Q20

${ }^{*}$ We thank Emily Breza and John Ledyard for helpful comments and suggestions.

${ }^{\dagger}$ Department of Economics, Universidad de Los Andes (jccarden@uniandes.edu.co).

${ }^{\ddagger}$ Department of Economics, Universidad de Los Andes (ca.mantilla967@uniandes.edu.co).

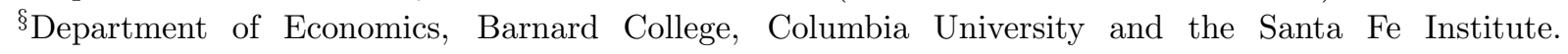
(rs328@columbia.edu). 


\section{Introduction}

Common property, as distinct from open access, is a regime involving a well-defined set of users, each of whom has rights to the extraction of an economically valuable resource. Many inshore fisheries, grazing lands, forest areas, and water resources are accessed under these conditions. The notion that such resources could be exploited in a manner that is sustainable and reasonably efficient is supported by numerous case studies, as well as theoretical models and experimental tests using subjects in the lab and the field. ${ }^{1}$

Experimental studies of common pool resource games have generally followed the protocol introduced by Ostrom et al. $(1992,1994)$. These experiments involve a finite number of rounds in which subjects take actions intended to mimic the extraction of a resource held as common property. This involves a negative externality, since higher levels of extraction reduce the marginal returns to extractive effort of all players. Standard equilibrium analysis predicts over-extraction of the resource relative to efficient levels under these conditions.

The behavior of experimental subjects typically deviates substantially from this equilibrium prediction in a number of interesting respects. All available actions are chosen with positive frequency, with strictly dominated actions being chosen persistently and often. The frequency distribution has a monotonic structure with higher levels of extraction, which are less individually costly, being selected more often. Average extraction is relatively stable over time, lying below equilibrium levels but above efficient levels.

We argue in this paper that these patters cannot be adequately explained by existing theories of other-regarding preferences or social norms and propose an alternative model that appears to fit the data well. This alternative is based on the notion of sampling equilibrium, introduced by Osborne and Rubinstein (1998). The basic idea underlying a sampling equilibrium is that individuals try out multiple actions, observe payoffs, and subsequently adopt actions that were the most rewarding. A sampling equilibrium is then a distribution of actions in a population that reproduces itself in the sense that the likelihood with which that action is taken matches the probability with which it is the most rewarding under the sampling procedure.

As demonstrated by Osborne and Rubinstein, a sampling equilibrium can involve the play of strictly dominated strategies with positive probability. However, every pure strategy Nash

\footnotetext{
${ }^{1}$ See Ostrom (1990) for theory and evidence, Bromley (1992) for a collection of case studies, Ostrom et al. (1992, 1994) for early experimental tests, Cardenas (2011) for more recent field experiments, and Sethi and Somanathan (1996) for an evolutionary argument.
} 
equilibrium is also a sampling equilibrium, so the model does not predict that dominated actions must be played with positive probability. To obtain this stronger claim, one can use a refinement of sampling equilibrium based on dynamic stability (Sethi, 2000). This refinement can select a unique sampling equilibrium in many games, and this happens to be the case in the prototypical common pool resource game. Significantly, the unique stable sampling equilibrium in such games involves the play of dominated strategies with positive probability, and implies a monotonic frequency distribution very much like those observed in the data.

We establish these claims by examining data from a variety of experimental studies, all involving common pool resource games. These include the classic studies of Ostrom et al. (1994), as well as more recent implementations with different payoff specifications. We find a striking congruence between predicted and observed frequency distributions in all implementations that involve student populations, although there is some bias towards more efficient actions relative to predictions based on stable sampling. For non-traditional subject populations involving individuals who need to manage common pool resources effectively in daily life, however, predictions based on sampling match the data less well. This is because individuals from these latter populations manage to obtain greater efficiency in resource use relative to student populations.

Our work contributes to a large literature on theoretical models of experimental behavior. One strand of this literature develops the idea that the material payoffs faced by experimental subjects do not accurately reflect their objective functions because they care about the entire payoff distribution, and perhaps also because their utilities are belief dependent as in the theory of psychological games. ${ }^{2}$ A second class of models, especially well suited to the analysis of repeated games, relies on reinforcement or other forms of real-time learning. ${ }^{3}$

These two distinct approaches have been merged in recent work by Arifovic and Ledyard (2012), who embed social preferences in a learning model with the goal of accounting for the basic stylized facts emerging from public goods experiments. Social preferences are introduced by allowing for individuals to care about both efficiency and envy, where the former is represented by average payoffs and the latter by any shortfall in one's own payoff

\footnotetext{
${ }^{2}$ Specific forms of other-regarding preferences capturing concerns for fairness and reciprocity have been proposed by Levine (1998), Fehr and Schmidt (1999), Bolton and Ockenfels (2000), Charness and Rabin (2002), and Cox et al. (2007). Belief-dependent utilities play a central role in Rabin (1993), Dufwenberg and Kirchsteiger (2004), and Falk and Fischbacher (2006), all of whom employ the theoretical construct of psychological games (Geanakoplos et al. 1989).

${ }^{3}$ See Erev and Roth (1998), Roth and Erev (1995), Hanaki et al. (2005), and Arifovic et al. (2006) for examples.
} 
relative to the average. Learning is modeled as follows. At each period there is a finite list of remembered actions from which choices are made in accordance with a probability measure. The items on this list need not be unique: a given action can have multiple replicates. Replication in each period takes place on the basis of hypothetical performance in the immediately preceding period, with an action that would have performed poorly being replaced by one that would have done better. This measure of hypothetical performance also determines which action is played in any given period, with higher valued actions being selected with greater likelihood. In addition, the list of remembered actions itself changes over time, as incumbent items are replaced by novel ones with some probability.

The approach taken in the present paper disregards social preferences entirely, and our model of stable sampling equilibrium is parameter free. In contrast, the Arifovic and Ledyard model has six free parameters. The parameter independence of our model is both a strength and a weakness. We are able to replicate certain aspects of observed behavior with high precision without imposing any parameter restrictions at all, which we view as a positive feature of the model. However, this comes at a cost, since the data do indicate that social preferences matter, and especially so for populations whose livelihoods depend on the effective management of common pool resources. In principle, an extension of the stable sampling equilibrium framework to allow for social preferences should be a straightforward task, and one that seems worth exploring in future research.

The rest of the paper is organized as follows. Section 2 introduces the basic structure of a common pool resource game and takes a first look at the data from one experimental implementation. The concept of sampling equilibrium is then introduced in Section 3 , together with the refinement that sharpens the predictions of the model. We examine the congruence between predicted and actual frequency distributions for student data in Section 4 , and consider the data from villagers with common property management experience in 5 . Section 7 concludes.

\section{Experimental Common Pool Resource Games}

The basic structure of a common pool resource game is as follows. There are $n$ players, each of whom faces a set of ordered actions $\left\{e_{1}, e_{1}+1, \ldots, e_{m}\right\}$, interpreted as levels of resource extraction. It is typically assumed that $e_{1}$ is either 0 or 1 . The action chosen by player $i$ is 
denoted $x_{i}$, and the aggregate extraction level is

$$
X=\sum_{j=1}^{n} x_{j}
$$

The payoffs to each player depend only on her own action and the aggregate action, and may be written

$$
\pi_{i}=g\left(x_{i}, X\right)
$$

The function $g$ is increasing in the first argument and decreasing in the second. That is, given a level of aggregate extraction, those with higher individual extraction levels get higher payoffs, but an increase in one person's extraction level lowers the payoff of all others. This external effect results in a divergence between individual incentives and collective interests, and equilibria with inefficiently high levels of extraction under standard assumptions.

Various versions of this game have been studied experimentally, following the pioneering work of Ostrom et al. $(1992,1994)$. There are five aspects of behavior that emerge consistently in this set of experiments: all available actions are chosen with non-negligible frequency in the subject population as a whole, more individually costly actions are chosen with lower frequency, most subjects choose a variety of strategies over the ten rounds, the average level of extraction within groups is stable over time and intermediate between efficient and equilibrium levels, and the heterogeneity in action choice does no appear to diminish over the rounds. We shall argue that the concept of stable sampling equilibrium accounts for this set of stylized facts.

As motivation for the model, we begin by describing the findings from one implementation of a common pool resource experiment (Cárdenas, 2004) and discuss the data from other studies in Section 4. Consider the following payoff function:

$$
\pi_{i}=a x_{i}-\frac{b x_{i}^{2}}{2}+\phi\left(n e_{m}-X\right),
$$

where $a, b$, and $\phi$ are positive constants. The first two terms reflect private benefits and costs arising from one's extraction level, while the third term captures the negative effect of higher aggregate extraction on each individual's payoff.

As long as $n \phi>a$, aggregate payoffs are maximized when all individuals $i$ choose the lowest extraction level $x_{i}=1$, and any symmetric action profile other than this is Paretodominated. In addition, if $a>\phi$ and $b$ is sufficiently small, then equilibrium extraction levels will be inefficiently high. Experimental parameters were set as follows: $a=60, b=5$, $\phi=20, n=5$ and $e_{m}=8$. Under these conditions the choice of $x_{i}=8$ is a dominant strategy, while social surplus is maximized if $x_{i}=1$ is chosen by all players. The social 
dilemma then appears in its starkest form: efficiency demands minimum extraction while maximum extraction is a dominant strategy.

Each session consisted of ten rounds, during which all participants had access to a payoff table based on the function (1); see Table A.1 in the Appendix. Individuals chose extraction levels (columns of the table) simultaneously in each of the rounds. At the end of each round, once all choices had been made, the aggregate extraction level was computed and announced publicly. Given this information, each subject was able to deduce her individual payoff from the table, by looking at the corresponding row and the chosen column. This was the only communication received by the subjects after each round. ${ }^{4}$

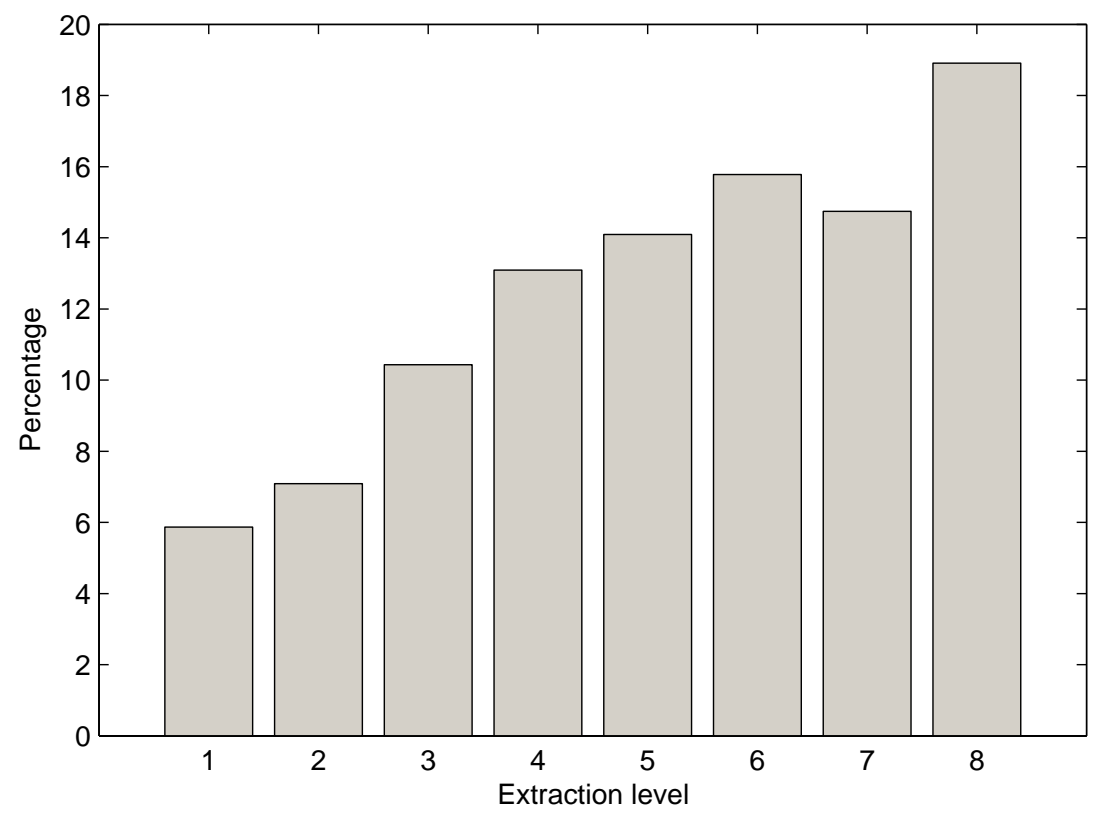

Figure 1: Distribution of strategies for the student sample in Cárdenas (2004)

Based on this protocol, Cárdenas (2004) reports results from two subject populations: students and villagers. ${ }^{5}$ The latter set of participants are drawn from communities in which management of resources held as common property is a routine and economically important

\footnotetext{
${ }^{4}$ Many of the experiments described here and in Section 4 had a second stage designed to uncover the effects of different institutions: one-shot and repeated communication, pecuniary and non-pecuniary penalties and endogenous rule-making. Although the first stage provides a baseline for the analysis of the effects of institutions introduced in the second stage, it also constitutes an independent source of analysis of human behavior in absence of coordination mechanisms. As we show below, there is remarkable consistency across experimental studies in the first-stage data.

${ }^{5}$ Experiments based on the same payoff function are also reported in Rodriguez et al. (2008) and Cárdenas (2011).
} 
activity. Here we present the student data, and defer discussion of the field data to Section 5 .

Figure 1 displays the distribution of strategies aggregated by participants and rounds. In general, higher extraction levels are chosen with greater frequency, and the dominant action (maximum extraction) is chosen about one-fifth of the time. These aggregates mask considerable variation in action choice at the individual level across rounds: about $70 \%$ of subjects chose maximum extraction at least once, while less than $1 \%$ chose this action in every round.

The considerable variability across rounds in actions chosen by given individuals is revealed in Figure 2. A negligible proportion of individuals limited themselves to a single action. About $88 \%$ of subjects chose at least four distinct actions across the ten rounds, and about $72 \%$ chose at least five. This suggests considerable experimentation on the part of subjects.

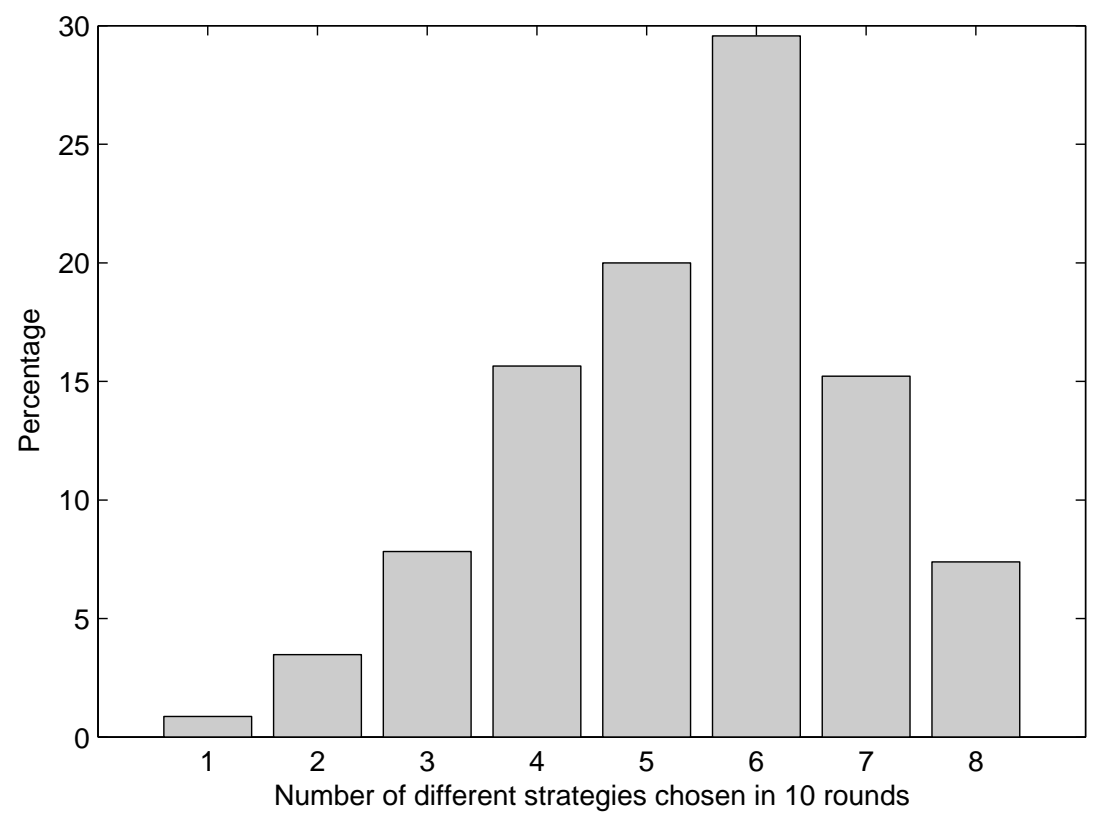

Figure 2: Different choices used by a player in 10 rounds

Next consider the manner in which the average action choice varies over rounds, shown in Figure 3. We see neither a convergence to the equilibrium action, nor to the efficient action. In fact, the average extraction level remains quite steady at an intermediate level.

It is conceivable that the intermediate extraction level seen in Figure 3 reflects convergence to an equilibrium of a different game, obtained by transforming the material payoffs 
in the table to allow for social preferences. Models of inequality aversion, for instance could predict intermediate extraction levels with suitably calibrated parameters. Alternatively, it is possible that the intermediate extraction level seen in the figure corresponds to a norm that restrains pure self-interest and promotes efficiency, but not to the extent of attaining welfare maximization of the group.

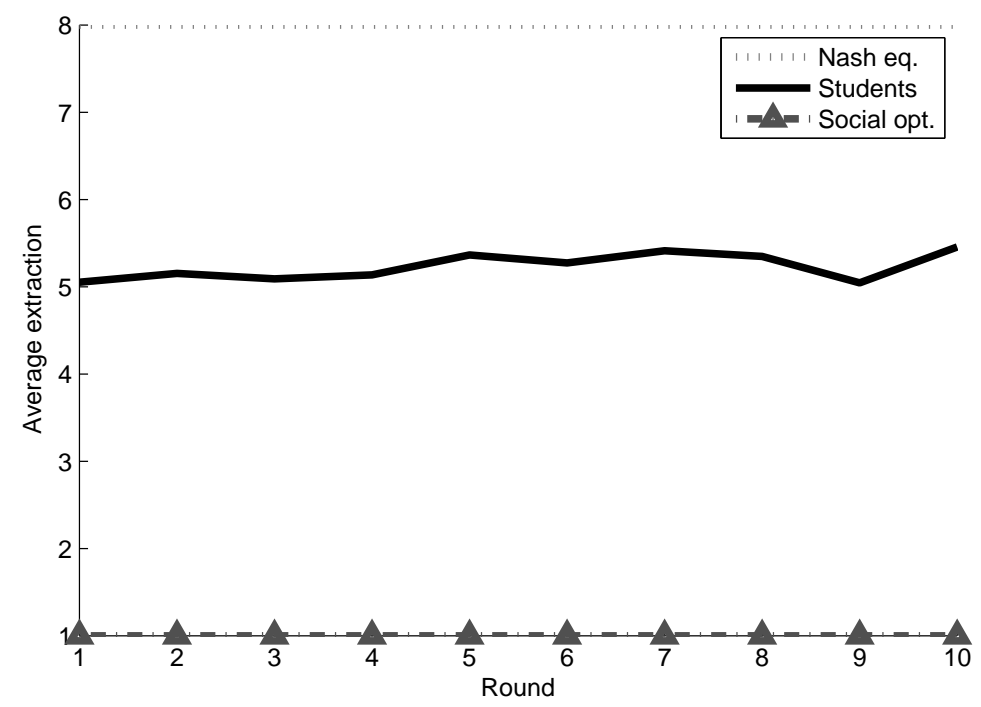

Figure 3: Average level of extraction over time

However, each of these explanations would require a decline in the variability of actions within groups as coordination on a social preference equilibrium or norm guided focal point were attained. This is not what we see in the data. Figure 4 shows that the within-group variance does not seem converge to zero, or even to diminish systematically over time. The left panel corresponds to the dynamics of the within-group standard deviation for the first ten rounds for the complete sample. We observe that the average standard deviation per group per round lies between 1.6 and 2.6 units throughout. To get a sense of what these magnitudes mean, note that a standard deviation of 1.6 would obtain if the the five players chose five different but consecutive actions. Higher values are obtained if the actions are even further dispersed: the choices $\{1,4,5,6,7\}$ would generate a standard deviation of 2.3 , still below the upper bound in the data.

To rule out the possibility that ten rounds are too few for convergence to obtain, the right panel of the figure shows data for three sessions in which interaction occurred over twenty rounds rather than ten. Again we see that that the variance of actions is maintained at a roughly constant level throughout.

The distribution of actions chosen by participants and the stability over time of both the 

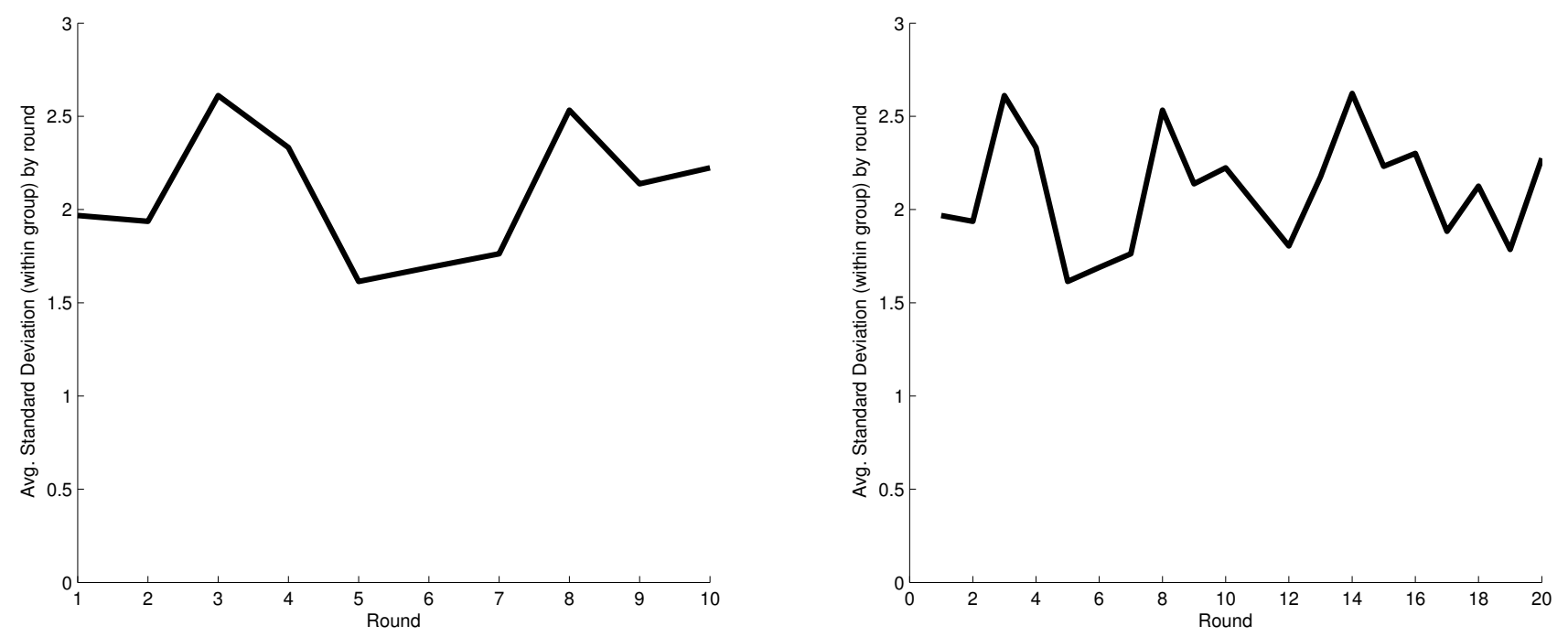

Figure 4: Average standard deviation (by group) over time

mean and the variance of this distribution suggest that conventional models based on social preferences cannot fully account for the data. In particular, the heterogeneity of actions at the level of an individual is suggestive of sampling and action selection in response to observed payoffs. We next describe a model of sampling equilibrium and a refinement based on dynamic stability that together predict a distribution of actions with full support and monotonic frequencies very much like that seen in Figure 1.

\section{Sampling Equilibrium}

The key idea underlying the concept of sampling equilibrium is that when faced with a novel strategic situation, individuals will experiment with actions, observe outcomes, and choose among available choices based on realized payoffs. The problem of selecting an action is viewed, in effect, as a multi-armed bandit problem in which a given probability distribution over the actions of others determines the consequences of any given choice by the subject in question. A sampling equilibrium is a distribution which is self-generating, in the sense that the probability of selecting an action conditional on this distribution matches the probability with which this action is taken in the distribution.

Formally, consider a symmetric game with actions $\left\{e_{1}, \ldots, e_{m}\right\}$, and let $\pi=\left(\pi_{1}, \ldots, \pi_{m}\right)$ denote some arbitrary probability distribution over these actions. Now suppose that a player samples each of the actions exactly once and, on each such trial, her opponent plays the 
mixed strategy given by $\pi$. Once all actions have been sampled, each will be associated with a realized payoff. Suppose that the player who is sampling selects the action with the highest realized payoff, breaking ties with uniform probability. Let $w_{i}(\pi)$ denote the probability that action $e_{i}$ is selected under this procedure. One interpretation of $w_{i}(\pi)$ is that it denotes the probability that action $e_{i}$ is selected when one's opponent at each step of the sampling procedure is independently drawn from a large population in which a proportion $\pi_{i}$ always play $e_{i}$.

A sampling equilibrium is simply a mixed strategy $\pi^{*}$ that satisfies

$$
w_{i}\left(\pi^{*}\right)=\pi_{i}^{*}
$$

for all $i$. That is, for each action $e_{i}$, the likelihood that it will be selected is equal to the likelihood with which is it currently being played. This may be interpreted as a steady state of a dynamic process in which a large incumbent population is choosing actions in the proportions $\pi_{1}, \ldots, \pi_{n}$, while new entrants choose actions in accordance with $w_{1}(\pi), \ldots, w_{n}(\pi)$. Such a dynamic process is implicit in the justification for sampling equilibrium offered by Osborne and Rubinstein, and requires that the rates of change $\dot{\pi}_{i}$ satisfy

$$
\dot{\pi}_{i}>0 \text { if and only if } w_{i}(\pi)>\pi_{i} .
$$

Stability with respect to this dynamic process can be used as a criterion for selection among multiple sampling equilibria.

The set of sampling equilibria can be large. It is easily seen, for instance, that every strict Nash equilibrium is also a sampling equilibrium. But sampling equilibria can also be surprising, and involve the choice of actions that are strictly dominated. In fact, this can occur even in sampling equilibria that are stable with respect to the dynamics (2). The following example, taken from Osborne and Rubinstein (1998), illustrates.

Consider the two player, three action symmetric game with payoffs as follows. Each individual has three actions, $\{1,2,3\}$, with payoff matrix given by

$$
\left(\begin{array}{lll}
6 & 3 & 0 \\
7 & 4 & 1 \\
8 & 5 & 2
\end{array}\right) .
$$

There is a dominant strategy equilibrium at $(0,0,1)$ which is also a sampling equilibrium and yields each player a payoff of 2 . But there is a second sampling equilibrium at $(0.20,0.28,0.52)$ in which both strictly dominated actions are played with positive probability. It turns out that only the latter equilibrium is stable under the dynamics (2). In fact, the basin of 
attraction of this equilibrium is the entire unit simplex, excluding the single point $(0,0,1)$. That is, trajectories from any initial state $\pi \neq(0,0,1)$ converge to the sampling equilibrium in which dominated actions are played with positive probability.

To get some intuition for why this is the case, consider an initial state $\pi=(\varepsilon, \varepsilon, 1-$ $2 \varepsilon$ ). We shall show that if $\varepsilon$ is sufficiently small, so the initial state is close to the unique Nash equilibrium, trajectories move away from this equilibrium. That is, the unique Nash equilibrium of the game is dynamically unstable. To see this, consider the events under which the third action will be selected under the sampling procedure when one's opponent at each stage is independently drawn from a population playing in accordance with $\pi$. There are three such mutually exclusive events: (i) the opponent takes action 3 in all three samples, (ii) the opponent takes action 2 when action 3 is sampled, and does not take action 1 when either action 1 or action 2 are sampled, and (iii) the opponent takes action 1 when action 3 is sampled. Summing the probabilities of these three events, we obtain

$$
w_{3}(x)=(1-2 \varepsilon)^{3}+\varepsilon(1-\varepsilon)^{2}+\varepsilon .
$$

Expanding this expression and collecting terms yields

$$
w_{3}(x)=1-4 \varepsilon+o\left(\varepsilon^{2}\right)
$$

where $o\left(\varepsilon^{2}\right)$ represents terms that are of order $\varepsilon^{2}$ or higher. Hence for $\varepsilon$ sufficiently small, $w_{3}(x)<1-2 \varepsilon=x_{3}$, so $\dot{x}_{3}<1$. The sampling dynamics result in a movement away from the dominant strategy equilibrium. ${ }^{6}$

Figure 5 depicts the unique stable sampling equilibrium of this game. Two features of this equilibrium are notable. All strategies, including those that are strictly dominated are played with positive probability. And the probabilities increase monotonically, with less individually costly actions being played with higher probability.

One might conjecture that stable sampling equilibria have the same structure for public goods games more generally. This is only partially true: the characteristics of stable sampling equilibria depend on the number of players and details of the payoff function. Strictly dominated actions cannot be played in a stable sampling equilibrium of a game unless the sum of the number of players and the number of actions is at least five. ${ }^{7}$ For public goods games

\footnotetext{
${ }^{6}$ This argument does not depend on the dominated actions being played with the same probability in the initial state. For a proof of the claim that that the sampling equilibrium in which dominated strategies are played with positive probability is the unique stable equilibrium, see Sethi (2000).

${ }^{7}$ Hence the unique stable sampling equilibrium in the prisoners' dilemma is simply the dominant strategy equilibrium.
} 


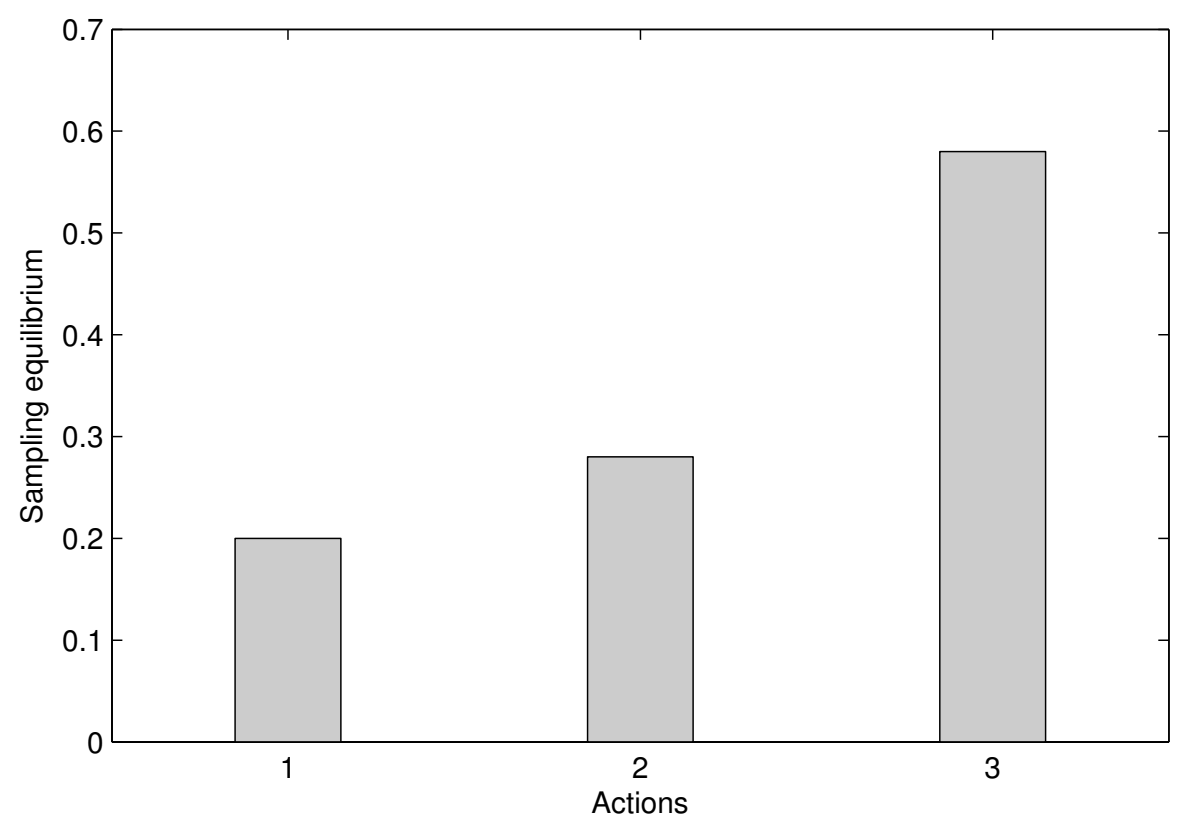

Figure 5: The unique stable sampling equilibrium for the $2 \times 3$ public goods game.

with many players and actions, the payoff function matters. Specifically, if the efficiency gains from contribution are too low, then the dominant strategy equilibrium is also the unique stable sampling equilibrium. Otherwise the frequency distribution has a monotonic structure very much like that in Figure 5.8

The same exercise can be carried out for the common pool resource game with payoff function (1), used in the Cárdenas (2004) experiments. Again we find two sampling equilibria, one of which is the dominant strategy equilibrium while the other has full support. The latter equilibrium is shown in Figure 6. As in the case of the above example, the dominant strategy equilibrium is unstable under the sampling dynamics, while the equilibrium depicted in the figure is stable. All trajectories originating at a disequilbrium point converge to the unique stable equilibrium depicted in the figure.

The qualitative features of the theoretically predicted distribution in Figure 6 bear a striking resemblance to the empirical distribution of actions shown in Figure 1. In both cases all available actions are chosen with positive probability, and less individually costly actions are chosen more frequently. The dominant action is chosen $24 \%$ of the time in the theoretical distribution, and $20 \%$ of the time in the data. There is some bias towards more

\footnotetext{
${ }^{8}$ Figure A.1 in the appendix shows the unique stable sampling equilibria for three public goods games with different payoff functions: in two of the three cases dominated actions are played with positive probability.
} 


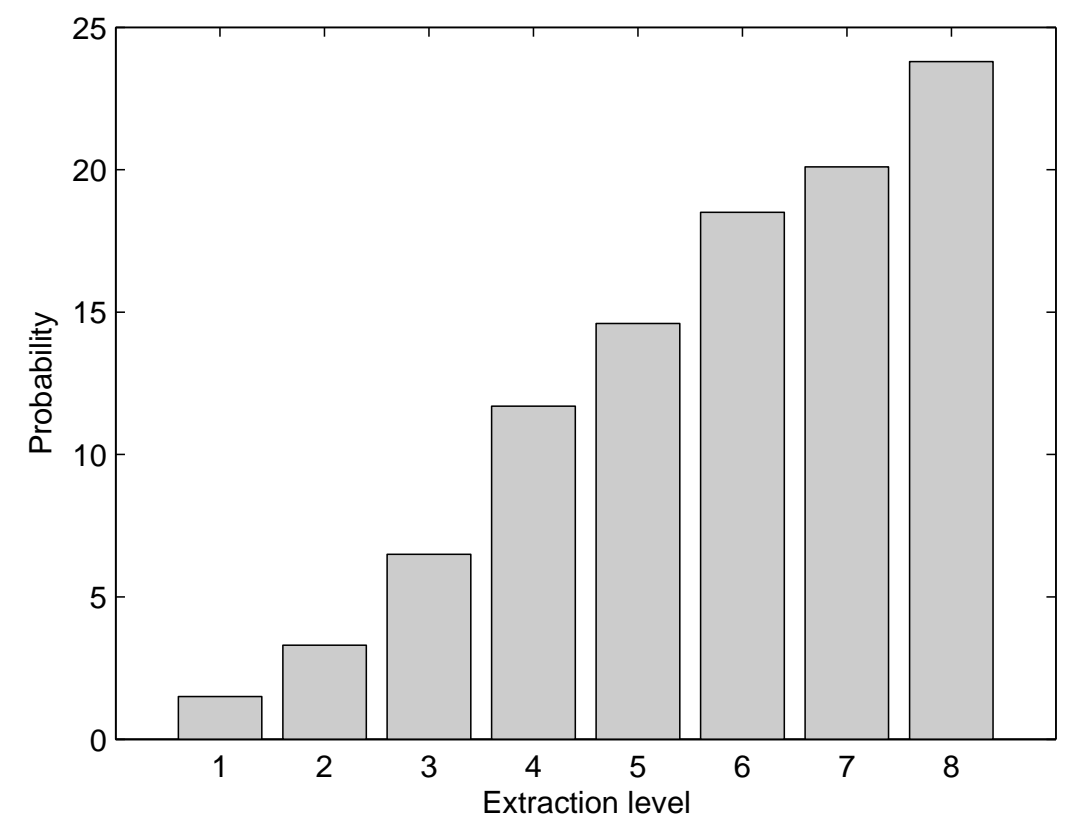

Figure 6: The unique stable sampling equilibrium for the $5 \times 8 \mathrm{CPR}$ game.

efficient outcomes relative to the prediction of the stable sampling equilibrium. In particular, the efficient action is chosen less than $2 \%$ of the time in the predicted distribution, but almost $6 \%$ of the time in the data.

We next show that this degree of consistency between theoretical and empirical frequencies arises in a number of other experiments.

\section{Predicticted and Observed Extraction Levels}

The predicted and observed extraction levels for the data discussed in Section 2 are shown in Figure 7. (This simply combines information from Figures 6 and Figure 1 into a single picture.) The qualitative features of the two distributions are similar, but the systematic bias towards more efficient actions is clear. The empirical distribution is flatter than the predicted distribution: for low extraction levels we observe realized frequencies larger than the predicted probabilities, while for high extraction levels we have the reverse. These differences suggest that other-regarding preferences and social norms also play a role in the determination of behavior.

Very similar patterns arise when one compares predicted and observed outcomes using 


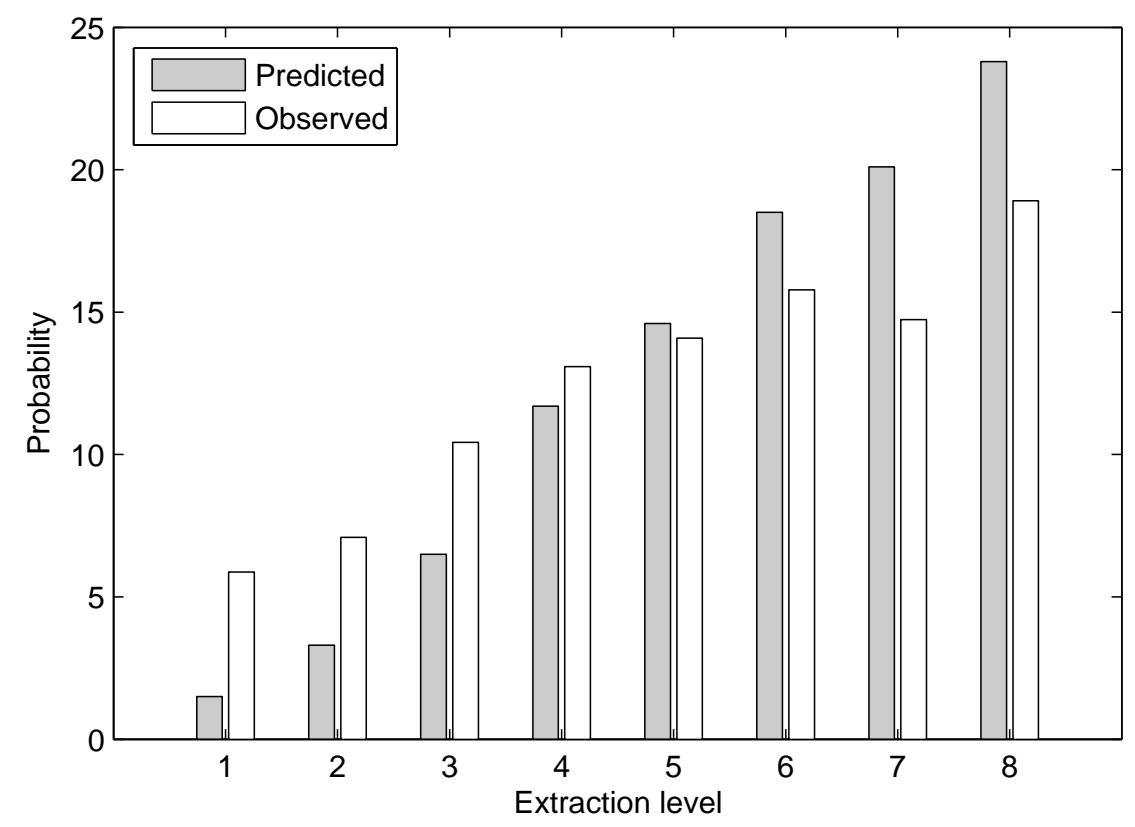

Figure 7: Predicted (sampling equilibrium) and realized distributions

data from other experiments. First consider the design used by Ostrom et al. (1994). Eight participants, each endowed with 10 tokens, had to divide these between two markets: one with a fixed rate of return and another with a rate dependent on the total amount invested by the group. With $x_{i} \in\{0, \ldots, 10\}$ denoting the amount invested in the latter market, the payoff to individual $i$ and action profile $x$ was:

$$
\pi_{i}(x)=w\left(e-x_{i}\right)+\frac{x_{i}}{X} f(X)
$$

if $X>0$ and $\pi_{i}(x)=$ we otherwise. The function $f$, representing total output from the common pool resource, was given by

$$
f(X)=0.23 X-0.0025 X^{2},
$$

and the fixed rate of return from the outside option was set at $w=0.05$. The unique Nash equilibrium action of this game is $x_{i}=8$, while aggregate payoffs are maximized if $X=36$, corresponding to average extraction is $x_{i}=4.5$. Hence both equilibrium and efficient extraction levels are interior.

The experiments involved 56 participants divided into seven groups. In each group, subjects interacted initially for 10 rounds without any kind of communication, receiving feedback only about the aggregate extraction level after each round. The realized frequency distribution is shown in Figure 8, together with the prediction based on the unique stable sampling equilibrium for this particular set of payoffs. 


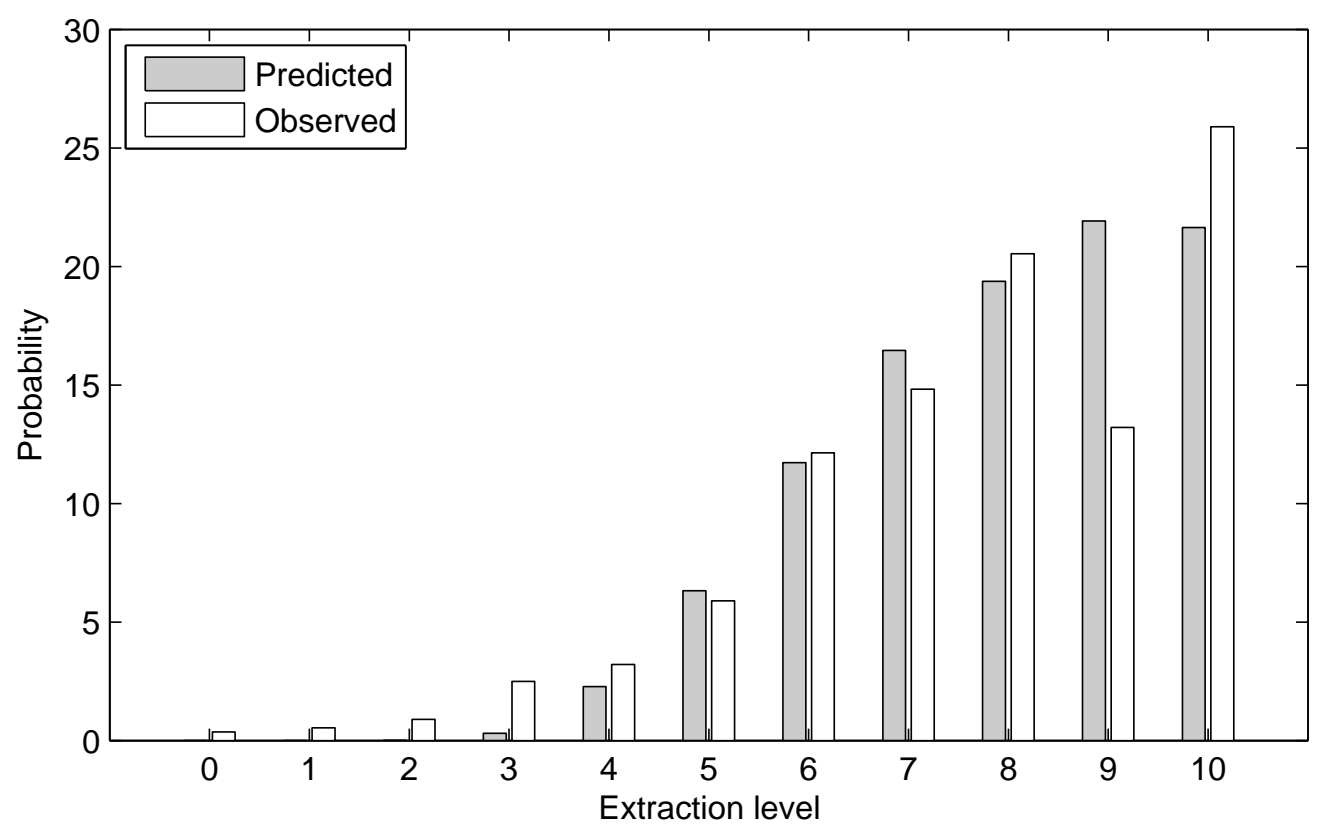

Figure 8: Predicted and Observed Extraction Frequencies for Ostrom et al. (1994)

For this specification the sampling equilibrium predicts that the lowest three extraction levels $\{0,1,2\}$ will not be chosen with positive frequency. Then the predicted frequencies rise monotonically from close to zero to $22 \%$ for extraction level 9 , before declining slightly at the highest extraction level. Comparing predicted with realized frequencies, we again find broad qualitative agreement. The lowest three extraction levels are each chosen less than $1 \%$ of the time, and frequencies rise monotonically thereafter, with the exception of a decline at extraction level 9 .

Finally consider the results reported in Carpenter and Cárdenas (2011) for a common pool resource game with 8 players, 9 extraction levels $\{0, \ldots, 8\}$, and following payoff function (originally used in Cárdenas et al., 2000):

$$
\pi_{i}(x)=k\left[\left(q-\frac{X^{2}}{2}\right)+\left(\gamma x_{i}-\frac{\phi}{2} X^{2}\right)+w\left(e-x_{i}\right)\right]^{\eta} .
$$

Parameters were set as follows: $w=30, \gamma=97.2, \phi=3.2, q=1372.8 ; k=2.38 \times 10^{-4}$ and $\eta=2$. In this case the Nash equilibrium extraction levels are $x_{i}=6$, while aggregate payoffs are maximized when and $x_{i}=1$. As in Ostrom et al. (1994), both efficient and equilibrium extraction levels are interior.

Experimental data are available for five sessions with 24 participants in each, for a total of 120 subjects, all college students. In each session participants were divided into three groups 


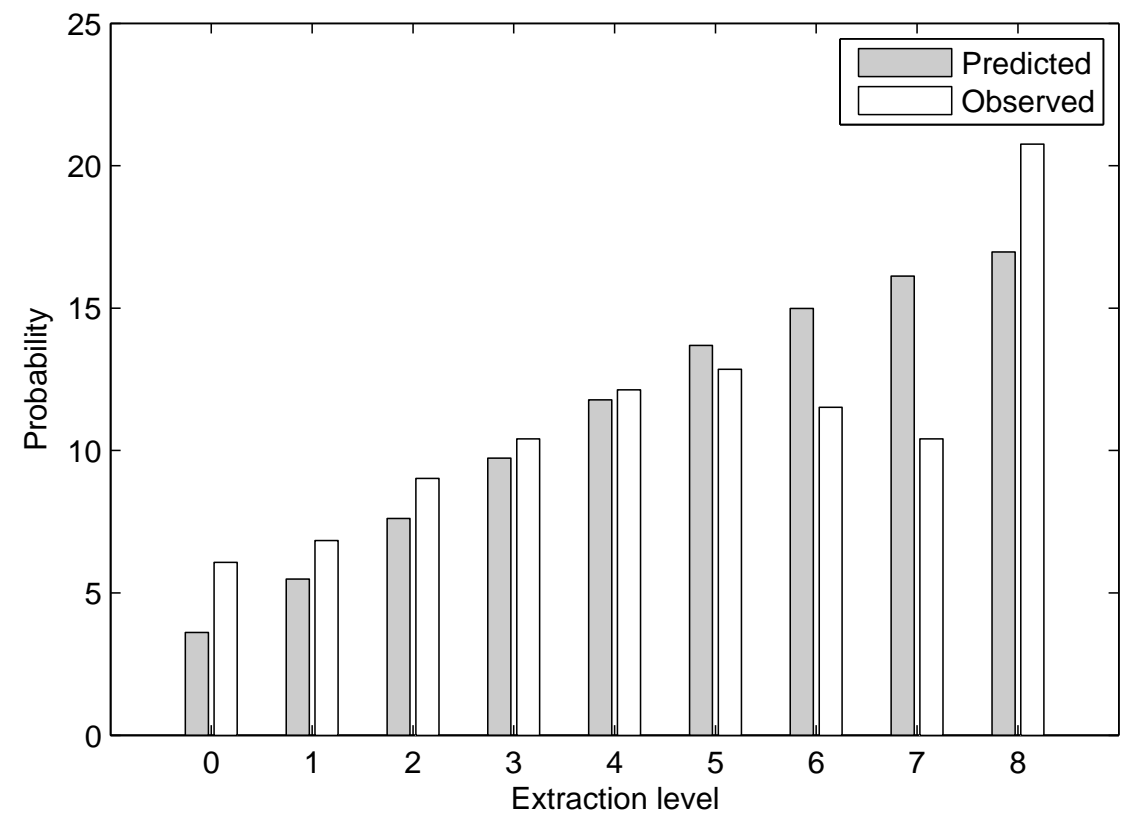

Figure 9: Predicted and Observed Extraction Frequencies for Carpenter and Cárdenas (2011)

of eight. Extraction decisions were made over 15 rounds, generating 1,800 observations. Observed and predicted frequencies for each extraction level are displayed in Figure 9, again using the unique stable sampling equilibrium as the basis for prediction. The predicted frequencies increase monotonically at all extraction levels, and observed frequencies follow the same pattern with the exception of extraction levels 6-7. Recall that this effect arose also in the Ostrom et al. (1994) data, and it appears that individuals choosing high levels of extraction are inclined to choose the highest available. This is, in fact, a myopic best response at all action profiles. Nevertheless, this action is chosen just $21 \%$ of the time, compared to the sampling equilibrium prediction of $17 \% .^{9}$

To summarize, qualitative predictions based on the concept of a stable sampling equilibrium fit data on common pool resource games conducted with student participants across a range of implementations. We turn next to data from the field-lab, with subjects drawn from populations whose livelihoods depend on the effective management of economically valuable resources held as common property.

\footnotetext{
${ }^{9}$ Furthermore, changes over time in the within-group standard deviation of actions is similar across the three experiments; see Figure A.2 in the appendix.
} 


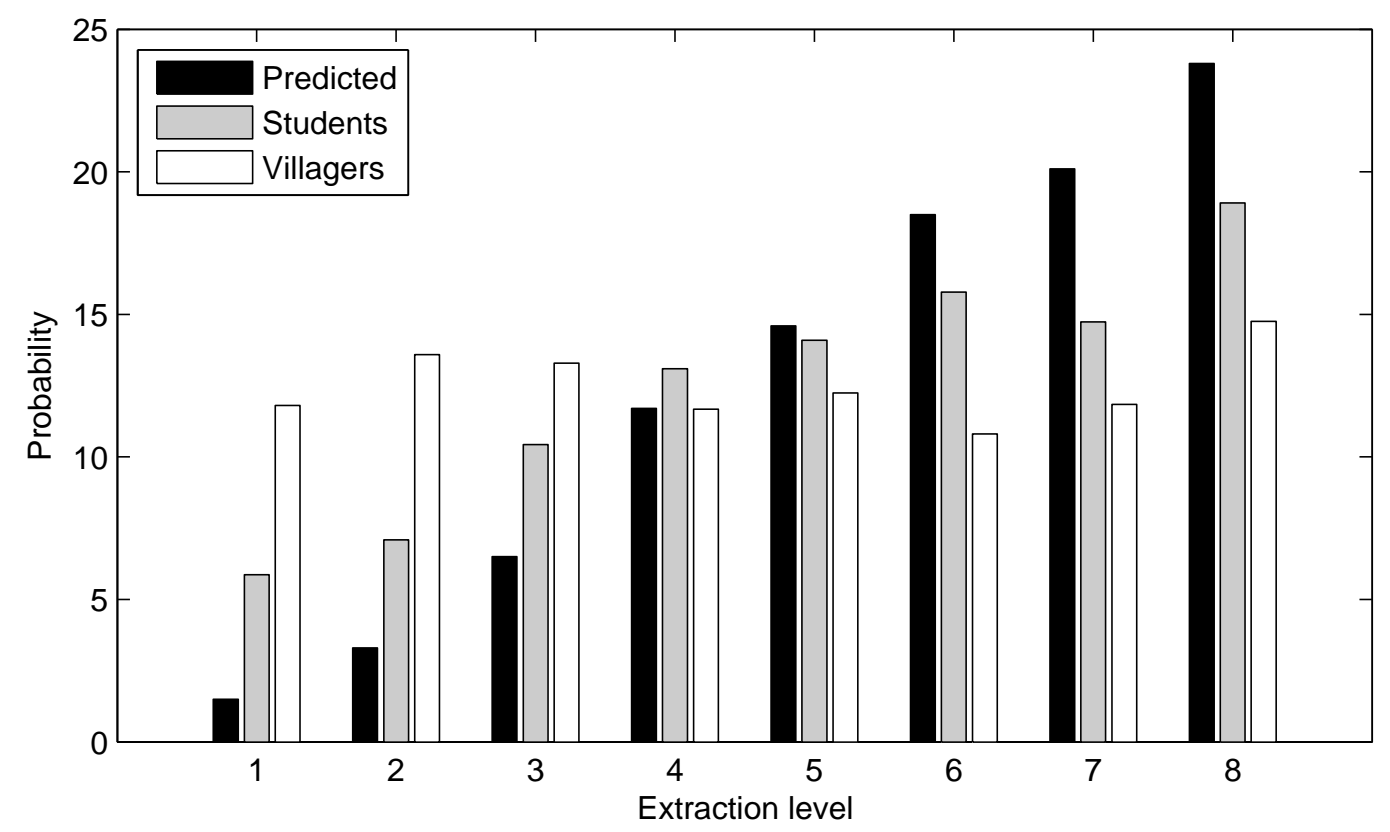

Figure 10: Predicted (sampling equilibrium) and realized distributions for separate samples of villagers and students

\section{Evidence from the Field-Lab}

The experiment described in Section 2 was implemented with two distinct subject pools. The data from urban students has already been discussed, and we now examine the data from a population of villagers for whom common pool resource management is a routine activity.

Figure 10 shows the frequency distribution for the both subject populations, alongside the predictions based on the unique stable sampling equilibrium for this game. As noted above, there is a close correspondence between the student data and the predicted frequencies, although subjects appear to choose somewhat lower extraction levels (and hence achieve slightly improved efficiency) relative to the sampling equilibrium.

This effect is considerably more pronounced in the population of villagers. Although all extraction levels are chosen with positive frequency by both groups, villagers are less likely to choose the dominant strategy, and more likely to choose efficient extraction. The dominant action was chosen $15 \%$ of the time by villagers and $19 \%$ of the time by students. One-third of villagers never choose this action at any round. In contrast, the socially optimal action was chosen $12 \%$ of the time by villagers, about twice as often as it was selceted by students. 
Average extraction by villagers was maintained at around 4.5 units, while the within-group standard deviation was held between 1.7 and 2.3. As with the student data, there is little sign of convergence to a single extraction level. Furthermore, there is little difference across the two subject pools in the number of different actions chosen by individuals: $74 \%$ of villagers and $72 \%$ of students chose at least five distinct actions over the course of the ten rounds.

These results suggest that social preferences or norms of restraint play a greater role among villagers than they do among the pool of urban students, mitigating the effects of sampling dynamics. Put differently, any explanation of behavior based on sampling needs to take into account the fact that subjects have other-regarding preferences, and that these may differ systematically across different populations.

\section{$6 \quad$ Statistical Tests}

The visual correspondence between observed and predicted extraction levels is striking, but falls short of a statistical test of the model. We now turn to more a more formal analysis of the match between theory and data.

Table 1 summarizes some of the key features of the four experimental designs, including Nash equilibrium and efficient per-capita extraction levels. It also provides some descriptive statistics. The average extraction in all cases lies between the efficient and equilibrium levels, and shows no sign of increasing or diminishing over the time. The within group standard deviation of actions also appears to be stable, and the mean number of actions samples is large relative to the cardinality of the action set in all cases.

\begin{tabular}{lcccc}
\hline & $\begin{array}{c}\text { Cárdenas (2004): } \\
\text { students }\end{array}$ & $\begin{array}{c}\text { Ostrom, Gardner } \\
\text { and Walker (1994) }\end{array}$ & $\begin{array}{c}\text { Carpenter and } \\
\text { Cárdenas (2011) }\end{array}$ & $\begin{array}{c}\text { Cárdenas (2004): } \\
\text { villagers }\end{array}$ \\
\hline Number of subjects & 230 & 56 & 120 & 705 \\
Subjects per group & 5 & 8 & 8 & 5 \\
Number of rounds & 10 & 10 & 10 & 10 \\
Action set & $\{1, \ldots, 8\}$ & $\{0, \ldots, 10\}$ & $\{0, \ldots, 8\}$ & $\{1, \ldots, 8\}$ \\
Nash equilibrium & 8 & 8 & 1 & 8 \\
Efficient per-capita extraction & 1 & 7.5 & 4.53 & 1 \\
Avg. extraction level: Rounds 1-5 & 5.16 & 7.86 & 4.71 & 4.60 \\
Avg. extraction level: Rounds 6-10 & 5.31 & 2.11 & 2.41 & 4.45 \\
Std. dev. (within groups): Rounds 1-5 & 1.96 & 1.93 & 2.48 & 2.08 \\
Std. dev. (within groups): Rounds 6-10 & 1.94 & 4.93 & 4.76 & 2.14 \\
Actions sampled in 10 rounds (mean) & 5.37 & & & 5.49 \\
\hline
\end{tabular}

Table 1: Summary Statistics for the four experiments 
Table 2 reports pairwise correlations between observed and predicted frequencies at each level of resource extraction. For all three student samples the correlations are statistically significant, and for the first two columns they are extremely high. This is the case despite substantial differences in payoff functions, resulting in a boundary equilibrium in one case and interior equilibria in the other two. The villager data shows no correlation at all, however, since observed extraction levels were much more uniformly distributed and hence biased towards efficient levels. These findings are consistent with the visual impression obtained in Figures $7-10$.

\begin{tabular}{ccccc}
\hline & $\begin{array}{c}\text { Cárdenas (2004): } \\
\text { students }\end{array}$ & $\begin{array}{c}\text { Ostrom, Gardner } \\
\text { and Walker (1994) }\end{array}$ & $\begin{array}{c}\text { Carpenter and } \\
\text { Cárdenas (2011) }\end{array}$ & $\begin{array}{c}\text { Cárdenas (2004): } \\
\text { villagers }\end{array}$ \\
\hline Correlation & $0.9749^{* * *}$ & $0.9375^{* * *}$ & $0.7924^{* *}$ & 0.0454 \\
& $(0.0000)$ & $(0.0000)$ & $(0.0109)$ & $(0.9150)$ \\
\hline$* * * \mathrm{p}<0.01,{ }^{* *} \mathrm{p}<0.05, * \mathrm{p}<0.1$ & & &
\end{tabular}

Table 2: Pearson correlation between realized and predicted distributions

Table 3 reports results from a regression of observed versus predicted frequencies. Here the prediction is a slope not different from unity, and lower slopes are indicative of a bias towards efficiency. We easily reject the hypothesis that the slopes equal zero for the three student experiments, though not for the one with villagers. Furthermore, we cannot reject the hypothesis that the slope is 1 for the Ostrom et al. (1994) and Carpenter and Cárdenas (2011) data, although this test has little power. All four point estimates for the slope are below 1, again suggesting that the omission of social preferences from our model comes at a cost.

\begin{tabular}{rcccc}
\hline & $\begin{array}{c}\text { Cárdenas (2004): } \\
\text { students }\end{array}$ & $\begin{array}{c}\text { Ostrom, Gardner } \\
\text { and Walker (1994) }\end{array}$ & $\begin{array}{c}\text { Carpenter and } \\
\text { Cárdenas }(2011)\end{array}$ & $\begin{array}{c}\text { Cárdenas (2004): } \\
\text { villagers }\end{array}$ \\
\hline Slope $\left(\beta_{1}\right)$ & $0.528^{* * *}$ & $0.887^{* * *}$ & $0.707^{* *}$ & 0.00709 \\
& $(0.0468)$ & $(0.153)$ & $(0.246)$ & $(0.0750)$ \\
Constant & $5.901^{* * *}$ & $1.025^{*}$ & 3.252 & $12.41^{* * *}$ \\
& $(0.640)$ & $(0.538)$ & $(1.830)$ & $(0.797)$ \\
Observations & 8 & 11 & 9 & 8 \\
R-squared & 0.950 & 0.879 & 0.628 & 0.002 \\
& & & & \\
Ho: $\beta_{1}=1$ & 101.76 & 1.42 & 0.54 & 175.23 \\
& $(0.0001)$ & $(0.2727)$ & $(0.4804)$ & $(0.0000)$ \\
\hline Robust standard errors in parentheses. ${ }^{* * *} \mathrm{p}<0.01, * * \mathrm{p}<0.05, * \mathrm{p}<0.1$ &
\end{tabular}

Table 3: OLS regression: Observed frequency versus predicted probability

Finally, we separately regress observed and predicted frequencies on the extraction level using a quadratic specification to allow for nonlinearities. This allows us to compare the 
ments appears as a consistent and striking feature of the data when one restricts attention to student subject pools.

We do not claim that the concept of stable sampling equilibrium alone can account for the experimental findings; it is clear that social preferences and norms also play a role, especially for populations with high dependence on common property. Our purpose, rather, has been to bring to the attention of experimental researchers a versatile theoretical construct that might be very effective in accounting for observed data when used in conjunction with other approaches.

Merging a theory of social preferences with sampling dynamics seems both tractable and worthwhile, although the resulting model will clearly not be parameter-free. Furthermore, the concept of stable sampling dynamics is versatile and can be applied to any normal form game. Testing predictions based on this hypothesis in other settings is accordingly an interesting and potentially fruitful area for future research. 


\section{A Appendix}

\begin{tabular}{|c|c|c|c|c|c|c|c|c|c|c|}
\hline & \multicolumn{8}{|c|}{ My level of extraction } & \multirow[b]{2}{*}{$\begin{array}{c}\text { Average } \\
\text { extraction } \\
\text { from others }\end{array}$} \\
\hline & & 1 & 2 & 3 & 4 & 5 & 6 & 7 & 8 & \\
\hline \multirow{29}{*}{ 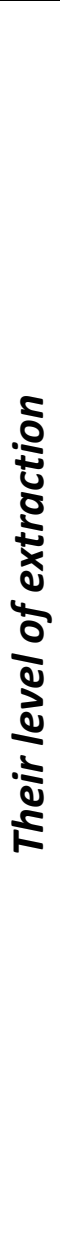 } & 4 & 758 & 790 & 818 & 840 & 858 & 870 & 878 & 880 & 1 \\
\hline & 5 & 738 & 770 & 798 & 820 & 838 & 850 & 858 & 860 & 1 \\
\hline & 6 & 718 & 750 & 778 & 800 & 818 & 830 & 838 & 840 & 2 \\
\hline & 7 & 698 & 730 & 758 & 780 & 798 & 810 & 818 & 820 & 2 \\
\hline & 8 & 678 & 710 & 738 & 760 & 778 & 790 & 798 & 800 & 2 \\
\hline & 9 & 658 & 690 & 718 & 740 & 758 & 770 & 778 & 780 & 2 \\
\hline & 10 & 638 & 670 & 698 & 720 & 738 & 750 & 758 & 760 & 3 \\
\hline & 11 & 618 & 650 & 678 & 700 & 718 & 730 & 738 & 740 & 3 \\
\hline & 12 & 598 & 630 & 658 & 680 & 698 & 710 & 718 & 720 & 3 \\
\hline & 13 & 578 & 610 & 638 & 660 & 678 & 690 & 698 & 700 & 3 \\
\hline & 14 & 558 & 590 & 618 & 640 & 658 & 670 & 678 & 680 & 4 \\
\hline & 15 & 538 & 570 & 598 & 620 & 638 & 650 & 658 & 660 & 4 \\
\hline & 16 & 518 & 550 & 578 & 600 & 618 & 630 & 638 & 640 & 4 \\
\hline & 17 & 498 & 530 & 558 & 580 & 598 & 610 & 618 & 620 & 4 \\
\hline & 18 & 478 & 510 & 538 & 560 & 578 & 590 & 598 & 600 & 5 \\
\hline & 19 & 458 & 490 & 518 & 540 & 558 & 570 & 578 & 580 & 5 \\
\hline & 20 & 438 & 470 & 498 & 520 & 538 & 550 & 558 & 560 & 5 \\
\hline & 21 & 418 & 450 & 478 & 500 & 518 & 530 & 538 & 540 & 5 \\
\hline & 22 & 398 & 430 & 458 & 480 & 498 & 510 & 518 & 520 & 6 \\
\hline & 23 & 378 & 410 & 438 & 460 & 478 & 490 & 498 & 500 & 6 \\
\hline & 24 & 358 & 390 & 418 & 440 & 458 & 470 & 478 & 480 & 6 \\
\hline & 25 & 338 & 370 & 398 & 420 & 438 & 450 & 458 & 460 & 6 \\
\hline & 26 & 318 & 350 & 378 & 400 & 418 & 430 & 438 & 440 & 7 \\
\hline & 27 & 298 & 330 & 358 & 380 & 398 & 410 & 418 & 420 & 7 \\
\hline & 28 & 278 & 310 & 338 & 360 & 378 & 390 & 398 & 400 & 7 \\
\hline & 29 & 258 & 290 & 318 & 340 & 358 & 370 & 378 & 380 & 7 \\
\hline & 30 & 238 & 270 & 298 & 320 & 338 & 350 & 358 & 360 & 8 \\
\hline & 31 & 218 & 250 & 278 & 300 & 318 & 330 & 338 & 340 & 8 \\
\hline & 32 & 198 & 230 & 258 & 280 & 298 & 310 & 318 & 320 & 8 \\
\hline
\end{tabular}

Table A.1: Payoff table 


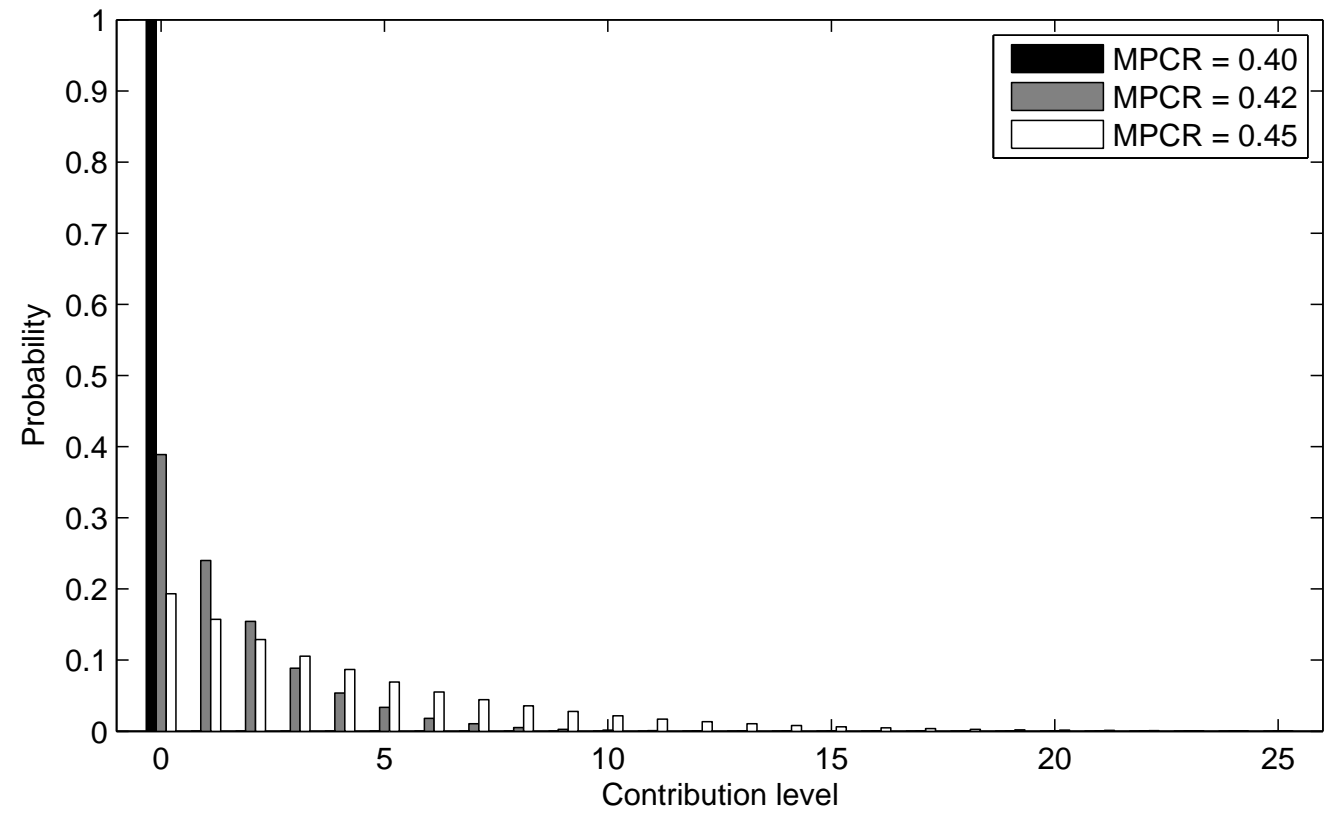

Figure A.1: Sampling equilibrium for Voluntary Contribution Mechanisms (VCM) with five players, an endowment of 25 tokens to invest in the private or in the common fund, and three different levels for the MPCR 

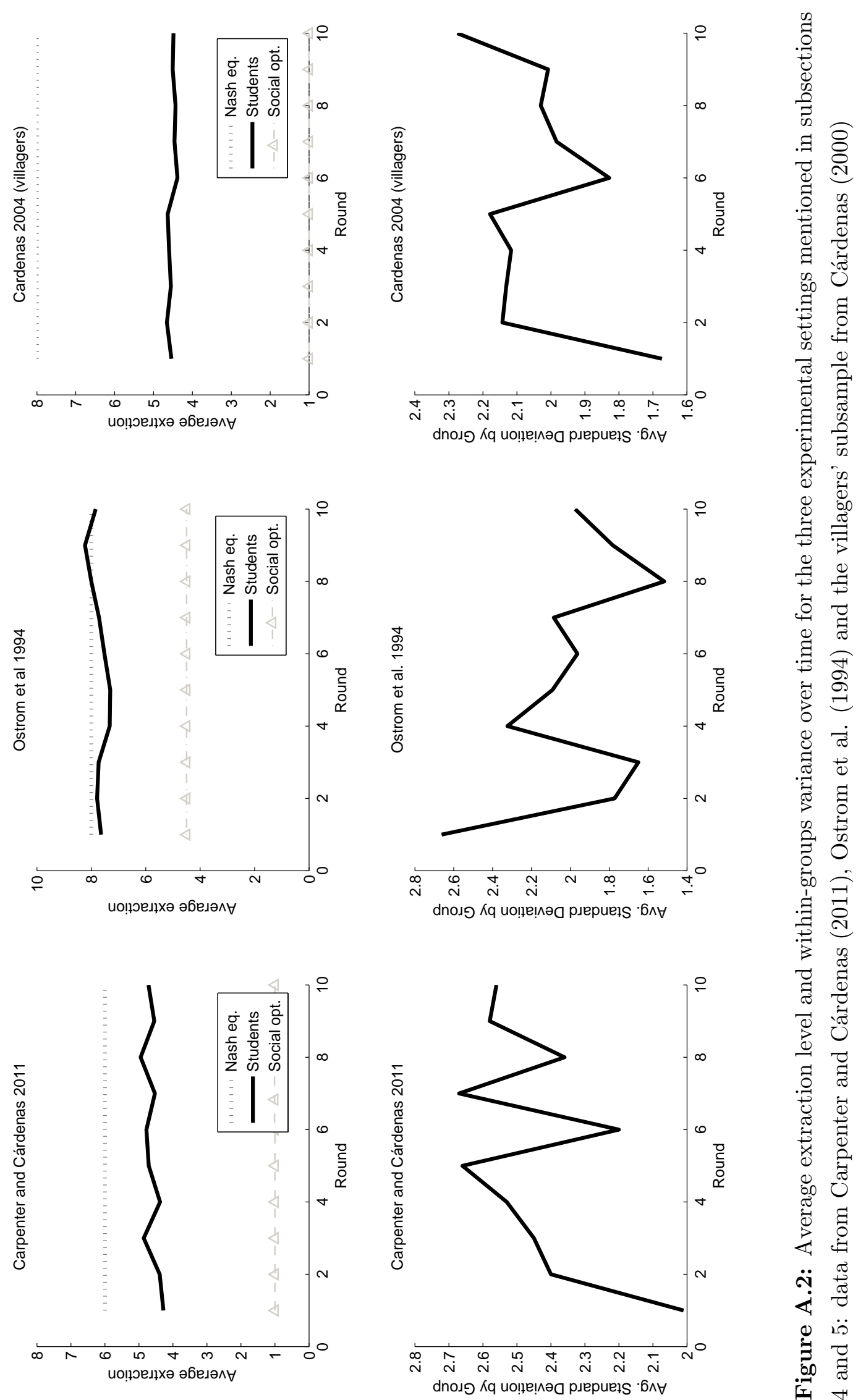


\section{References}

[1] Arifovic, Jasmina and Ledyard, John (2012). "Individual evolutionary learning, otherregarding preferences, and the voluntary contributions mechanism." Journal of Public Economics 96(9-10): 808-823

[2] Arifovic, Jasmina, Richard McKelvey and Svetlana Pevnitskaya (2006). "An initial implementation of the Turing tournament to learning in repeated two-person games." Games and Economic Behavior 57(1): 93-122

[3] Axelrod, Robert (1984). "The Evolution of Cooperation." Basic Books. New York.

[4] Bolton, Gary and Ockenfels, Axel (2000). "A Theory of Equity, Reciprocity and Competition." The American Economic Review 90(1): 166-193

[5] Bromley, D.W. (1992). "The Commons, Property and Common Property Regimes". pp.315. In: Making the Commons Works Theory, Practice and Policy. D.W. Bromley (ed.), Institute of Contemporary Studies, San Francisco.

[6] Bowles, Samuel and Gintis, Herb (2011). "A Cooperative Species: Human Reciprocity and its Evolution." Princeton University Press

[7] Cárdenas, Juan Camilo, John Strandlund and Cleve Willis (2000). "Local environmental control and institutional crowding-out." World Development 28(10): 1719-1733

[8] Cárdenas, Juan Camilo (2011). "Social Norms and Behavior in the Local Commons as Seen Through the Lens of Field Experiments." Environmental Resource Economics 48: $451-485$

[9] Carpenter, Jeffrey and Cárdenas, Juan Camilo (2011). "An Intercultural Examination of Cooperation in the Commons." Journal of Conflict Resolution 55(4): 632-651

[10] Charness, Gary and Rabin, Matthew (2002). "Understanding social preferences with simple tests." The Quarterly Journal of Economics 117(3): 817-869

[11] Cox, James C., Daniel Friedman and Steven Gjerstad (2007). "A tractable model of reciprocity and fairness." Games and Economic Behavior 59: 17-45

[12] Dufwenberg, Martin and Kirchsteiger, Georg (2004). "A theory of sequential reciprocity." Games and Economic Behavior 47(2): 268-298 
[13] Erev, Ido and Roth, Alvin (1998). "Predicting How People Play Games: Reinforcement Learning in Experimental Games with Unique, Mixed Strategy Equilibria." The American Economic Review

[14] Falk, Armin, Ernst Fehr and Fischbacher, Urs (2002). "Appropriating the Commons: A Theoretical Explanation." In E.Ostrom, T.Dietz, N.Dolsak, P.C. Stern, S. Stovich, and E.U.Weber (eds). The Drama of the Commons, National Research Council, National Academy Press.

[15] Falk, Armin and Fischbacher, Urs (2006). "A theory of reciprocity." Games and Economic Behavior 54: 293-315

[16] Fehr, Ernst and Schmidt, Klaus (1999). "A Theory of Fairness, Competition and Cooperation." Quarterly Journal of Economics 114(3): 817-868

[17] Geanakoplos, John, David Pearce and Ennio Stacchetti (1989). "Psychological Games and Sequential Rationality." Games and Economic Behavior 1: 60-79

[18] Hanaki, Nobuyukim, Rajiv Sethi, Ido Erev and Alexander Peterhansl (2005). "Learning strategies." Journal of Economic Behavior and Organization 56(4): 523-542

[19] Levine, David (1998). "Modelling Altruism and Spitefulness in Experiments." Review of Economic Dynamics 1: 593-622

[20] Osborne, Martin and Rubinstein, Ariel. (1998) "Games with Procedurally Rational Players." The American Economic Review 88: 834-847

[21] Ostrom, Elinor (1990). "Governing the Commons: the evolution of institutions for collective action" American Political Science Review 92(1): 1-22

[22] Ostrom, Elinor, Roy Gardner and James Walker (1994). "Rules, games and commonpool resources." University of Michigan Press, Ann Harbor

[23] Ostrom, Elinor, James Walker and Roy Gardner (1992). "Covenants With and Without a Sword: Self-Governance is Possible." American Political Science Review 86: 404-417

[24] Ostrom, Elinor (2006). "The value-added of laboratory experiments for the study of institutions and common-pool resources." Journal of Economic Behavior \& Organization. Vol. 61 (2006) 149163

[25] Poteete, Amy, Marco Janssen and Elinor Ostrom (2010). "Working Together: Collective Action, The Commons, and Multiple Methods in Practice". Princeton University Press, Princeton. 
[26] Rabin, Matthew. (1993) "Incorporating Fairness into Game Theory and Economics." The American Economic Review 83(5): 1281-1302

[27] Richerson, P.J., Robert Boyd and B. Paciotti (2002). "An Evolutionary Theory of Commons Management." In The Drama of the Commons. Ostrom, Elinor and Dietz, Thomas and Dolsak, Nives and Stern, Paul C. Stern and Stonich, Susan and Weber, Elke U., Eds. , National Academy Press, Washington, D.C.

[28] Rodríguez-Sickert, Carlos, Ricardo Andrés Guzmán and Juan Camilo Cárdenas (2008). "Institutions influence preferences: evidence from a common pool resource experiment". Journal of Economic Behavior and Organization 67(1): 215-227.

[29] Roth, Alvin and Erev, Ido (1995). "Learning in extensive-form games: Experimental data and simple dynamic models in the intermediate term." Games and Economic Behavior 8(1): 164-212

[30] Sethi, Rajiv. (2000) "Stability of Equilibria in Games with Procedurally Rational Players." Games and Economic Behavior 32: 85-104

[31] Sethi, Rajiv and Somanathan, E. (1996) "The Evolution of Social Norms in Common Property Resource Use." American Economic Review 86(4): 766-788 